\title{
Integrated chaotic communication scheme
}

\author{
Murilo S. Baptista \\ Institute of Physics, University of São Paulo, P.O. Box 66318, CEP 05315-970, São Paulo, São Paulo
}

Elbert E. Macau

INPE, Brazilian Institute for Space Research, P.O. Box 515, CEP 12227-010, São José dos Campos, São Paulo

Celso Grebogi

Institute for Plasma Research, Department of Mathematics, University of Maryland, College Park, Maryland 20742

Ying-Cheng Lai

Departments of Mathematics and Electrical Engineering, Arizona State University, Tempe, Arizona 85287-1804

Epaminondas Rosa, Jr.

Department of Physics, University of Miami, Coral Gables, Florida 33146

(Received 18 October 1999; revised manuscript received 2 May 2000)

\begin{abstract}
We present the characteristics and an analysis of a proposed communication scheme fully based on chaos theory. The key point is that the proposed scheme introduces the dynamical system as a way to encode and decode information and as a signal wave generator. In this scheme, all the protocols used to communicate digitally are fully integrated into one single design based on a chaotic modulation process. The chaotic encoder finds a set of trajectories that codes the information into a hard to decode chaotic wave form that carries a large amount of information. We also show how our scheme can handle multiplexing, which is also used as a way to enhance security, and its ability to handle noise.
\end{abstract}

PACS number(s): $05.45 . \mathrm{Vx}, 05.45 . \mathrm{Gg}, 01.20 .+\mathrm{x}$

\section{INTRODUCTION}

The idea of using chaos as a fundamental building block for constructing communication systems appeared in Ref. [1]. In that work, the authors manipulated a chaotic system, using arbitrarily small time-dependent perturbations, to generate controlled chaotic orbits whose symbolic representation corresponds to the digital representation of a desirable message. Subsequently, this idea was experimentally demonstrated in the scope of an electronic circuit, as reported in Ref. [2]. Recent works, aiming to implement more efficient chaotic-based communication systems, dealt with the synchronization of chaotic trajectories [3], noise filtering from noisy chaotic trajectories [4], chaotic error correcting codes [5], chaos coding [6], and cryptography with chaos [7].

The fundamental argument that has been emphasized about using chaotic-based communication system is its efficiency. In fact, a nonlinear chaotic oscillator that generates a wave form for transmission can be easily built, while all the electronics that is necessary for encoding the information in the chaotic signal remains as a low-power and inexpensive microelectronic circuit. However, so far, it appears that the full meaning of the word "efficiency" characterizing the use of chaos for communication is not well understood and fully appreciated.

The purpose of this paper is to show a communication system that uses chaos for performing the main tasks that are expected nowadays from a digital communication system. In fact, more than sending information through a communication channel, a digital communication system must also perform the following two fundamental functions: (i) source encoding, which compacts, compresses, and encrypts the source message; and (ii) channel encoding, which guarantees that the encoded message is robust against the presence of noise in the channel. Both operations encode one bit stream into another. In a standard digital communication scheme, each of these functions is not only accomplished by different subsystems, but, cumbersomely, the final sequence of bits must be modulated a posteriori into a wave form signal that can be adequately transmitted over a channel. This complex and involved scenario can be radically simplified if the communication system is based on chaos instead. All those functions and operations can be performed with the use of only one subsystem, the same one that performs the proper modulation of the signal for transmission over the communication channel. Thus we propose a communication system that inherits the most important advantages of both analog and digital communication systems: simplicity and efficiency (as in the digital system, our communication scheme can transmit information that is compacted, hard to decode, and robust against noise). This integrated communication scheme is attainable especially because a chaotic signal exhibits a kind of short memory that can be exploited to create a scheme where the coding and decoding protocols is not performed in the source but rather in the wave signal. So the signal is not only the carrier but also the message itself.

We now present our scenario of an integrated chaotic communication scheme which uses chaotic dynamics per se as a means to address standard communication issues such as encoding, noise reduction, compaction, and so on. For the sake of clarity, the results shown here are derived from a mapping. We assume that this mapping represents the dy- 
namics of a chaotic trajectory, obtained from a flow, in a Poincare section. Thus, from the discrete trajectory, there must exist a continuous trajectory that lies in a higherdimensional space connecting every point of this mapping in the corresponding section (suspension [8]). Thus, from now on, a chaotic wave signal is, in fact, a set of points obtained through a discreting process (a mapping) of the higherdimensional continuous trajectory, a trajectory which is the wave signal used to transmit information over a channel.

To find the coding trajectories on which information is sent, we could define a partition of the phase space as done in Ref. [2]. However, we would have to deal with symbolic forbidden transitions, a limitation that is typical in real chaotic dynamical systems. To overcome this limitation, we would need to implement an extra protocol in the communication scheme, a dynamical encoding, as done in Ref. [2], such that the message can be coded into a set of feasible trajectories. Instead, in this work, we look for a dynamical partition for which the coding trajectories, obtained from it, carry a large amount of information efficiently, are hard to decode, and are robust against channel noise. This is all achieved by using a new dynamical partition of the phase space, whose construction is based on the source message itself, and not on the dynamical system as in Ref. [1].

In traditional communication, to make the transmission robust against the presence of noise in the channel, a process that adds redundant information into the encoded source message used. In our proposed scheme, to make the transmission of chaotic signal robust against noise, the length of the coding trajectories must have a minimum length. The determination of the appropriate trajectory length is what we define to be the chaotic dynamical channel encoder. Therefore, we create wave signals that carry not only the message itself, but also the dynamic information from which the wave form, in the presence of dropouts and/or transmission noise, can be reconstructed. The length of each trajectory depends on some quantities that we will describe below.

This paper is organized as follows. In Sec. II, we present the proposed integrated scheme. In Sec. III, we describe how to implement this method by giving an example. In Sec. IV, we introduce the notion of entropy to compare this method with a traditional digital system. In Sec. V, we discuss some relevant properties of the proposed method regarding the changing of parameters to adjust the proposed system to function as aimed. In Sec. VI, we discuss the use of this method when a low noise level is present in the channel, and, in Sec. VII, we describe the implementation of a channel dynamical encoder which must be used when there is high noise level in the channel. Finally, in Sec. VIII, we make some general remarks regarding communication with chaos.

\section{PROPOSED INTEGRATED COMMUNICATING SCHEME}

In this section, we present our ideas by introducing the following communication system. We consider an information source that is modeled by a discrete-time random process $\left\{X_{i}\right\}_{i=-\infty}^{\infty}$, where all $X_{i}$ 's are independent and identically distributed random variables taking values on a discrete set. The source is, therefore, considered to be a discrete memoryless source (d.m.s), which means that it is a discrete- time discrete-amplitude random process. Let $\mathcal{S}$ $=\left\{s_{1}, s_{2}, \ldots, s_{N}\right\}$ be the alphabet set in which the random variable $X$ takes its values, and let the probability mass function for the discrete random variable $X$ be denoted by $p_{i}$ $=p\left(X=s_{i}\right)$ for all $i=1,2, \ldots, N$. We define as the message $M$ or the information sequence $M$ an ordered and finite sequence of outputs of the random variable $X$ which is to be transmitted between the source and the destination, or receiver, by using a communication system.

A communication system must perform the following tasks: (a) To convert the information sequence to another more efficient form of representation in order to transmit to the receiver. (b) To introduce redundancy in the information sequence that can be used at the receiver to overcome the effects of noise and other interferences faced by the signal during the transmission over the communication channel. (c) To map the message into a signal wave form that can be properly sent through the communication channel. (d) To recover at the receiver the information sequence that was generated at the source. In this paper, we consider a communication system that codes the information into a hard to break chaotic wave form, robust against noise, and carrying a large amount of information. Our communication system codifies the information sequence on chaotic trajectories of a flow. Furthermore, we assume that we can define a Poincare section transversal to this flow so that the system dynamics of the flow is well represented by a discrete map on this Poincaré section. We represent an iteration of this Poincare map as

$$
x_{i+1}=F\left(x_{i}\right) .
$$

Let us assume that we are given a typical message $M$ $=\left\{m_{0}, m_{1}, \ldots, m_{l-1}\right\}, m_{i} \in S$, where $l$ is large enough so that $M_{l}$ can be regarded as a good approximation of a typical sequence [9] of the information source that we are considering, and $l \geqslant N$. In fact, it is known that, for $l$ large enough, with a probability approaching 1 , every sequence from the source is a typical sequence, i.e., it has the same composition. Consider an arbitrary and fixed initial condition $x_{0}^{*}$ that is used to create a trajectory $T_{x_{0}^{*}}=\left\{x_{0}^{*}, x_{1}^{*}, \ldots, x_{l-1}^{*}\right\}$ of length $l$ as a result of $l-1$ iterations of the chaotic map $F$. The initial condition $x_{0}^{*}$ is not necessarily in the invariant chaotic set $A$. Let us introduce a fixed parameter $\epsilon$ so that, to each point $x_{i}^{*}$ of the trajectory we associate a hypercube $\beta\left(x_{i}^{*}, \epsilon\right)$ with center in $x_{i}^{*}$ and edge length $2 \epsilon$. Note that since $F$ is ergodic on $A$, the sequence $B\left(x_{0}^{*}, l, \epsilon\right)$ $=\left\{\beta\left(x_{0}^{*}, \epsilon\right), \beta\left(x_{1}^{*}, \epsilon\right), \ldots, \beta\left(x_{l-1}^{*}, \epsilon\right)\right\}$ covers part of $A$ for some $l>l_{l m}(\epsilon)$. Given $B\left(x_{0}^{*}, l, \epsilon\right)$, we consider a subsequence $B_{p}\left(x_{0}^{*}, l, \epsilon\right)$ of $B\left(x_{0}^{*}, l, \epsilon\right)$, so that $\beta\left(x_{i}^{*}, \epsilon\right)$ does not intersect the previous hypercubes $\beta\left(x_{j}^{*}, \epsilon\right), j<i$, in the subsequence, i.e., $\beta\left(x_{i}^{*}, \epsilon\right) \cap \beta\left(x_{j}^{*}, \epsilon\right)=\varnothing$, for $j<i$. Thus we have

$$
B_{p}\left(x_{0}^{*}, l, \epsilon\right)=\left\{\beta\left(x_{i_{1}}^{*}, \epsilon\right), \beta\left(x_{i_{2}}^{*}, \epsilon\right), \ldots, \beta\left(x_{i_{q}}^{*}, \epsilon\right)\right\},
$$

where one has $q \leqslant l-1$ for sufficiently small $\epsilon$. We introduce a parameter $r$ such that in the sequence $\left\{x_{i_{1}}^{*}, x_{i_{2}}^{*}, \ldots, x_{i_{q}}^{*}\right\}$ [the points that are located in the center of the hypercubes of the subsequence $\left.B_{p}\left(x_{0}^{*}, l, \epsilon\right)\right], F\left(x_{i_{k-1}^{*}}^{*}\right)=x_{i_{k}}^{*}$ for $(n-1) r$ 
$<k \leqslant(n) r$, with $(n=1,2,3, \ldots, q / r)$, but $x_{i_{(n r+1)}^{*}}^{*}$ is not necessarily the forward iteration of $x_{i_{n r}}^{*}$. Thus, if $r=2, i_{2}=i_{1}+1$, and $i_{4}=i_{3}$, by definition $i_{1}=0$, so that $\beta\left(x_{0}^{*}, \epsilon\right)$ corresponds to the letter $m_{0}$ of the message. We say that $B_{p}\left(x_{0}^{*}, l, \epsilon\right)$ creates a pseudopartition of part of $A$, which is associated with the units $s_{i}$ of the alphabet $S$. Now we associate the message $M$ with $B_{p}\left(x_{0}^{*}, l, \epsilon\right)$ by doing the following: for each $k$ from 1 to $q$, the hypercube $\beta\left(x_{i_{k}}^{*}, \epsilon\right)$ is associated to the letter $m_{k}$ of the message $M$. Note that at the end of this procedure we can have the unit $s_{i} \in S$ associated to many hypercubes of $B_{p}\left(x_{0}^{*}, l, \epsilon\right)$. This happens because the set $S$ has only $N$ units $s_{i}$, where $N \ll l$. Consequently, as a result of this process we have $N$ subsets $P_{i}$, one for each unit of $S$, where each $P_{i}$ is formed by the union of the hypercubes $\beta\left(x_{i_{k}}^{*}, \epsilon\right)$ that are associated with the specific unit $s_{i}$ of $S$. Thus we create a topological correspondence among the units of the alphabet and the disconnected regions of the invariant chaotic set $A$.

The preceding paragraph defines formally how to construct the pseudopartition. Such a description might be difficult to understand, so now we present a simple example on how to find such a pseudopartition, say, for $r=2$. Given the initial condition $x_{0}^{*}$, if $x_{1}^{*}=F\left(x_{0}^{*}\right)$ is such that $\beta\left(x_{1}^{*}, \epsilon\right)$ does not intersect the hypercube $\beta\left(x_{0}^{*}, \epsilon\right)$, we say that $\beta\left(x_{0}^{*}, \epsilon\right)$ belongs to the pseudopartition that encodes for the unit $s_{i}$, corresponding to the first letter of the message $M$, which is $m_{0}$, and $\beta\left(x_{1}^{*}, \epsilon\right)$ encodes for $m_{1}$. We note that in this case the indexes $i_{q}$ 's are $i_{1}=0$ and $i_{2}=1$. Then we iterate $x_{1}^{*}$, obtaining $x_{2}^{*}$. Now two cases must be considered: (1) if $x_{2}^{*}$ is such that $\beta\left(x_{2}^{*}, \epsilon\right)$ does not intersect the hypercubes $\beta\left(x_{0}^{*}, \epsilon\right)$ and $\beta\left(x_{1}^{*}, \epsilon\right)$, and $\beta\left(x_{3}^{*}, \epsilon\right)$ does not intersect all these previous hypercubes, from the previous iterations, then we say that $\beta\left(x_{2}^{*}, \epsilon\right)$ encodes for $m_{2}$, and $\beta\left(x_{3}^{*}, \epsilon\right)$ codes for $m_{3}$. (2) If either $\beta\left(x_{2}^{*}, \epsilon\right)$ or $\beta\left(x_{3}^{*}, \epsilon\right)$ intersects the previous hypercubes, then the hypercubes $\beta\left(x_{2}^{*}, \epsilon\right)$ or $\beta\left(x_{3}^{*}, \epsilon\right)$ are discarded, and we look for hypercubes $\beta\left(x_{n}^{*}, \epsilon\right)$ (for $n>3$ ) that code for the next two ( $r$ =2) elements of the message $M$, namely, $m_{4}$ and $m_{5}$, using the algorithm proposed in case (1). One might say that the letters of messages $m_{2}$ and $m_{3}$ will not be encoded by our method. We can, however, guarantee that for $\epsilon$ not too high, eventually the pair $\left(m_{2}, m_{3}\right)$ will be encoded by a pair of hypercubes $\beta\left(x_{i_{q}}^{*}, \epsilon\right)$ and $\beta\left(x_{i_{q+1}^{*}}^{*}, \epsilon\right)$, where $i_{q}$ will be different from 2 and $i_{q+1}$ will be different from 3 . We can also guarantee that, for $\epsilon$ not too high, each other pair $m_{k}$ and $m_{k+1}$ will also be encoded by a pair of hypercubes.

Considering this framework, we now explain how an arbitrary message $M=\left\{m_{0}, m_{1}, \ldots, m_{M-1}\right\}$ can be codified in a chaotic trajectory. Given this message, we decompose it into consecutive subsequences of $r$ symbols. The value of $r$, once chosen, remains the same during the operation of the communication system. Some major properties associated with communication systems depend on it, as it will be discussed below. Thus

$$
M=M_{0} \% M_{1} \% \quad \% M_{j-1},
$$

where \% stands for a concatenation operation, and $M_{0}$ $=\left\{m_{0}, \ldots, m_{r-1}\right\}, M_{2}=\left\{m_{r}, \ldots, m_{2 r-1}\right\}$, and so on. Note that all the subsequences have the same length. If the last subsequence does not have $r$ letters, dummy letters must be added. Suppose that the chaotic trajectory is initially at a point $x_{0}^{\prime}$. We take a unit that corresponds to the letter $m_{0}$, say $s_{j}$, and look in the subset $P_{j}$ for some hypercube $\beta\left(x_{i_{k}}, \epsilon\right)$ near $x_{0}^{\prime}$. The $r-1$ subsequent iterations of $x_{i_{k}}$ belong to hypercubes that are associated with the subsequent $r-1$ letters of $M_{1}$ in the same order. This procedure generates the trajectory $T_{1}$ that codifies the submessage $M_{1}$. We repeat the same procedure for $M_{2}$, this time having as the initial condition the $r$ th forward iteration of the point $x_{0}^{\prime}$. As a result of this concatenation procedure, trajectories $T_{i}$, each with $r$ points, are associated with each the $M_{i}$ submessages. The forward iteration of the last point of a given trajectory $T_{i}$ is near to the first point of the subsequent trajectory $T_{i+1}$. This means that small perturbations are enough to concatenate all the $i$ trajectories corresponding to the $i$ messages. Furthermore, for each trajectory $T_{i}$, just its initial condition is enough to generate all the subsequent $r-1$ points of the trajectory. In other words, for each submessage $M_{i}$, only the point associated with its first letter needs to be known. The other subsequent $r-1$ symbols of the message $M_{i}$ appear as a result of the natural dynamical evolution of the chaotic system with the proper initial condition.

To recover the message emitted by the source, the receiver needs to know the dynamical system and its parameters, the value of $r$, and the fixed initial condition $x_{0}^{*}$ which generates the $N$ subsets $P_{i}$ of the pseudopartitions. Each pseudopartition is associated with a unit $s_{i}$ of the alphabet $S$. The receiver decodes the message by identifying the chaotic trajectory with the available information provided by the pseudopartitions $P_{i}$. Furthermore, only the initial condition of each trajectory $T_{i}$ needs to be sent over the communication channel. This means, (and this is quite important) that for each set of $r$ symbols produced by the information source, only one value - the initial condition-needs to be sent over the communication channel in order to recover the message.

\section{EXAMPLE OF AN INTEGRATED COMMUNICATION SYSTEM}

To illustrate our communication method, we consider an information source to be a discrete memoryless source, as defined previously, which means that it is a discrete-time discrete-amplitude random process. Let $S=\left\{s_{1}, s_{2}, s_{3}, s_{4}\right\}$ be an alphabet set composed of four units on which the random variable $X$ takes its values, and let the probability mass function for the discrete random variable $X$ be denoted by $p_{i}$ $=p\left(X=s_{i}\right)$ with, say, the following values: $p_{1}=\frac{1}{2}, p_{2}=\frac{1}{4}$, $p_{3}=\frac{1}{8}$, and $p_{4}=\frac{1}{8}$. The chaotic mapping $F$ we use to codify the information sequence in its chaotic trajectories is the logistic map

$$
x_{i+1}=F\left(x_{i}\right)=b x_{i}\left(1.0-x_{i}\right),
$$

where we choose $b=4.0$. In this case, the hypercubes $\beta\left(x_{i}^{*}, \epsilon\right)$, generated from an arbitrary and fixed initial condition $x_{0}^{*}$, are the intervals $\left[x_{i}^{*}-\epsilon, x_{i}^{*}+\epsilon\right]$, where the parameter $\epsilon$ is fixed. Starting from a typical sequence $M_{l}$ of the information source with $l \geqslant N$, and considering an arbitrary 
